\title{
Maximization of Two Techniques of Relay Networks using Co-Operative Protocals
}

\author{
K.Shanmugapriya, D.Jayapriya, Kavitha G
}

\begin{abstract}
This study recognise the transfer-assisted co-usable transmission in remote systems, where numerous client pairs lead bidirectional interchanges through various transfers depending on the transmission of the Orthogonal Frequency Division Multiplexing. The fundamental goal is to improve the overall execution of the framework by increasing the overall performance. It manages the joint enhancement of channel and hand-off task, subcarrier portion just as hand-off determination. The issue is detailed as a combinatorial improvement issue. It primarily manages two-way transferring and to make it progressively manageable. It received a chart based methodology. Along these lines the issue is fathomed ideally in polynomial time by changing it into Maximum Weighted Bipartite Matching (MWBM) issue. The reproduction result shows the correlation of the proposed calculation and the seat mark alongside two handing-off conventions.
\end{abstract}

Keywords: Two way relaying, Bidirectional Communications, OFDM, subcarrier pairing, Graphical approach.

\section{INTRODUCTION}

Under high rate and consideration, the incorporation of hand-off assisted co-employable communication into the current cell structure is regarded as the most practical development. In assessment with legacy cell sort out move helped co-usable correspondence organize acknowledges relative inclinations over incorporation efficiency, action cost breaking point and transmission.

Regardless of the way that the correspondence is coordinated by move helped frameworks, it encounters mishap in frightful adequacy in light of the half duplex transmission in valuable systems. Starting late framework coding (by methods for bidirectional transmission mode decision) has indicated basic potential for improving framework throughput[3].Thus two way moving is familiar with improve supernatural profitability close by off helped bidirectional correspondence and it crushes the half duplex issue when stood out from the single heading relaying[1] [4].

Resource apportioning has pulled in wide thought starting late in a combination of OFDM based hand-off networks[5]-[10]. For the perfect exchange and subcarrier task in an OFDMA hand-off framework with various sources, different exchanges and a singular objective was investigated

Revised Manuscript Received on October 22, 2019.

* Correspondence Author

K.Shanmugapriya, Department of Computer Science and Engineering, Bharath Institute of Higher Education and Research, Chennai, Email: shanmugapriyabiher@gmail.com

D,Jayapriya, Department of Computer Science and Engineering, Bharath Institute of Higher Education and Research, Chennai. Email: priyajp8@gmail.com

Kavitha G, Department of Computer Science and Engineering, Bharath Institute of Higher Education and Research, Chennai, Email: kavithag90@gmail.com in [5]. In [7] the issue handles subject to twofold rot system . In [5][7] the works are normal that the exchange helped two bob transmission uses the identical subcarrier for uplink and downlink.

The crucial objective is to support the hard and fast throughput of the structure and it oversees two moving protocols. The giving off isn't continually basic in the hand-off helped correspondence [5]-[10],[15] (for instance direct associate correspondence) is accessible in the framework .

This paper is dealt with as seeks after: Section II introduces the structure model. In portion III ,Optimization layout work is explained. In portion IV Simulation results. Upcoming work is explained in secion VI

\section{SYSTEM MODEL}

Enlivened by the two way handing-off conventions, for example, The proposed transmission convention can without much of a stretch suit diverse transmission modes in a brought together manner.. As indicated by the channel conditions every client pair can choose any of the transmission modes. In direct transmission mode client pair can trade the data through the subcarriers legitimately with no utilization of transfers.

In this framework model, all hubs are dependent upon their very own individual pinnacle control limitation and along these lines, the transmit control is thought to be fixed and consistently disseminated. Let $\mathrm{N}=\{1,2, \ldots \mathrm{N}\}$ mean the arrangement of subcarriers, 


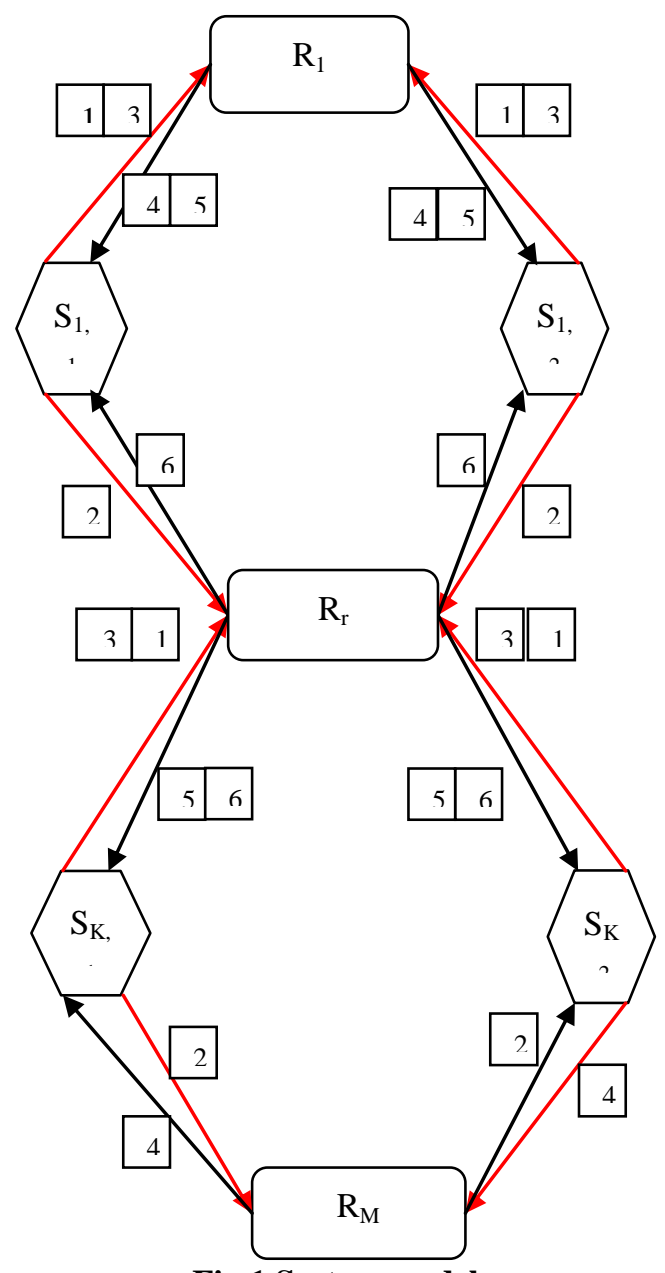

Fig 1.System model

\section{OPTIMIZATION FRAMEWORK}

In case the customer pair $k$ is doled out with subcarrier $n$ and sends sign to hand-off rin the essential stage, the hand-off $r$ then imparts the improved got banner on subcarrier $n^{\prime}$ in the $2^{\text {nd }}$ stage by the AF move show. Thusly by the DF hand-off show, the hand-off conveys the got sign by disentangling it on the subcarrier $n^{\prime}$ in the resulting stage

Direct Transmission: The achievable rate pair is viably procured as

$$
\begin{aligned}
& R_{M A C}=\frac{1}{2} \mathrm{C}\left(\gamma_{k 1 k 2}^{n n}\right) \\
& R_{B C}=\frac{1}{2} \mathrm{C}\left(\gamma_{k 2 k 1}^{n n}\right)---
\end{aligned}
$$

I to node $\mathrm{j}$ and assumes the noise variance of all nodes.

$$
R_{D}=\frac{1}{2} \mathrm{C}\left(\gamma_{k 1 k 2}\right)
$$

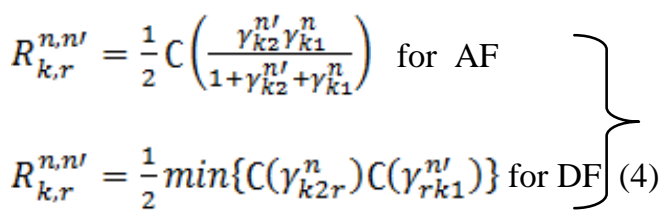

Two way moving : This is the 3-arrange two-path giving off, 1to hand-off R1 in the MAC stage ,and S1,2transmits its sign to hand-off R1, by then hand-off mixes the got banner and imparts it to both customer sets $\mathrm{S} 1,1$ and $\mathrm{S} 1,2$ as second organize BC. If AF or DF gave, the practicable rate sets are offered freely. The practicable total pace of customer pair $\mathrm{k}$ over subcarrier pair $\left(n, n^{\prime} \quad\right)$ with the support of AF hand-off

$$
\begin{aligned}
& R_{k_{1} r}^{n, n I}=\frac{1}{2} C\left(\frac{\gamma_{k_{1}, r}^{n} r_{r k_{2}}^{n I}}{1+v_{r k_{2}}^{n !}+v_{k_{1} r}^{n}+v_{k_{2} r}^{n}}\right)+ \\
& \frac{1}{2} C\left(\frac{v_{k_{2} r}^{n} r_{r k_{2}}^{n !}}{1+v_{r k_{1}}^{n !}+v_{k_{1} r}^{n} r+v_{k_{2} r}^{n}}\right) \\
& R_{k s}^{n, n}=\frac{1}{2} \min \left\{\mathrm{C}\left(\gamma_{k 1 r}^{n}\right) \mathrm{C}\left(\gamma_{r k 2}^{n \prime}\right) \mathrm{C}\left(\gamma_{k 2 r}^{n}\right)\right\}+ \\
& \frac{1}{2} \min \left\{\mathrm{C}\left(\gamma_{k 2 r}^{n}\right) \mathrm{C}\left(\gamma_{r k 1}^{n}\right) \mathrm{C}\left(\gamma_{r k 2}^{n}\right)\right\}
\end{aligned}
$$

$$
\begin{aligned}
& \text { P1: } \quad \max \sum_{k \in \mathcal{K}} \sum_{r \in \mathcal{M}} \sum_{n \in \mathcal{N}} \sum_{n^{\prime} \in \mathcal{N}} R_{k, r}^{n, n^{\prime}} \rho_{k, r}^{n, n^{\prime}} \\
& \text { s.t. }(2),(3) .
\end{aligned}
$$

\section{RESULTS DISCUSSION}

Imagine a 2D plane of focus districts appeared in Fig.3, hand-off focuses are abstractly in any case dependably dissipated in the relating square areas. The way in which misfortune model is obtained in [6], where the way in which fiasco model is set to 4 and Log-ordinary shadowing's standard deviation is set to $5.8 \mathrm{~dB}$. Subcarrier measurement is $\mathrm{N}=32$. All sources have similar, most absurd power destinations, as do all businesses

The fixed subcarrier blending plan is considered as a showcase benchmark $[3,5]$. Let the sign be sent by the client pair MAC sort out is sent by a hand-off in the BC mastermind to the virtually identical subcarrier, i.e. $\pi(n)=n$, rather than scanning for the ideal subcarrier blending. By then, the issue decreases in selecting the ideal client program and hand-off for enhancing the throughput for each subcarrier. The presentation of the proposed figure is separated from the Figure (3) and the standard near until AF appears and the presentation is separated from the Figure (4) along with the DF Protocol. 


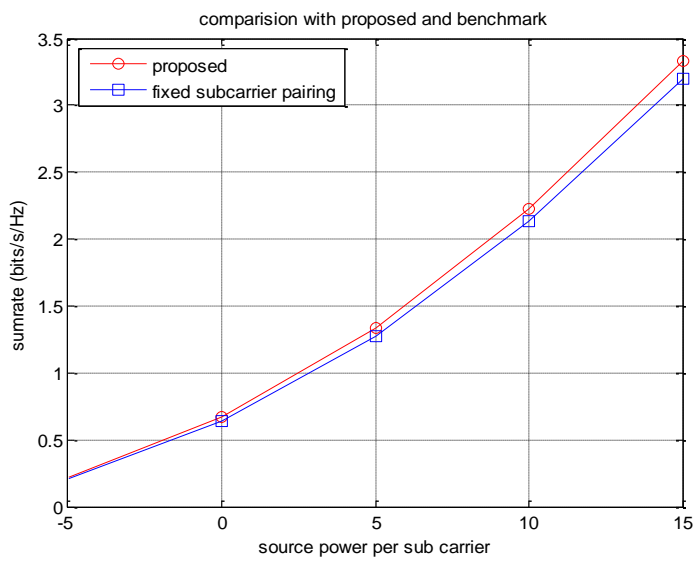

Fig. 3. Performance comparison of the proposed algorithm and the benchmark along with AF protocol.

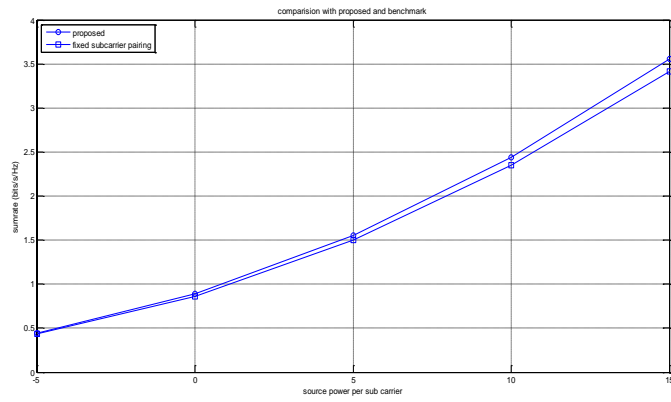

Fig 4. Proposed algorithm with DF protocol

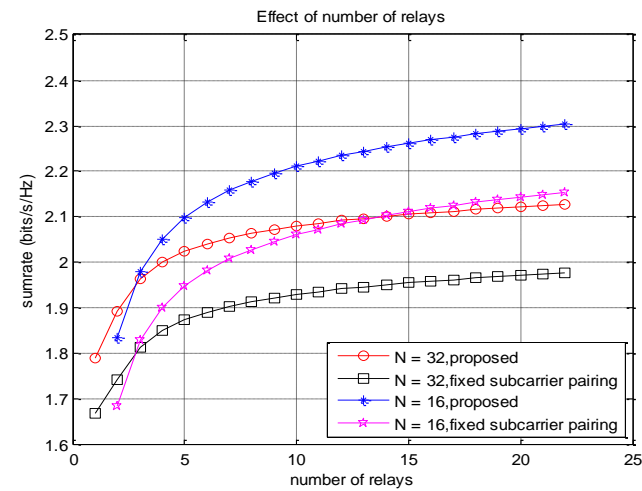

Fig 5. Effects of number of relays

\section{CONCLUSION AND FUTURE WORK}

In this work, the joint enhancement of subcarrier-based subcarrier-matching task and hand-off determination with $\mathrm{AF}$ and DF transfer convention for multi-transfer multi-pair two-way hand-off OFDM systems was explored. The problem has been described as a problem of combinatorial streamlining. We suggested a bipartite coordination approach for dealing with the issue in polynomial time preferably. Using diagrams is indicated the distinction between the proposed and the fixed plan. With the number of subcarrier adjustment, get the connection between the quantity of transfers used to the quantity of the transfers. This comparable issue depends on further developed two-way regenerative hand-off methodologies and can later be considered on works with different joining techniques.

\section{REFERENCES}

1. BAPATLA, D. and PRAKRIYA, S., 2019. Performance of a
Cooperative Network with an Energy Buffer-Aided Relay. IEEE Transactions on Green Communications and Networking, 3(3), pp. 774-788.

2. USHA, M. and RAMAKRISHNAN, B., 2019. A Robust Architecture of the OLSR Protocol for Channel Utilization and Optimized Transmission Using Minimal Multi Point Relay Selection in VANET. Wireless Personal Communications, 109(1), pp. 271-295.

3. DEVI, B.R., RAO, K.K. and RANI, M.A., 2019. Application of Modified Bellman-Ford Algorithm for Cooperative Communication. Wireless Personal Communications, 109(3), pp. 2025-2049.

4. NGUYENA, T.N., TRANC, M., DUY-HUNG, H., TRANGE, T.T and VOZNAK, M., 2019. Multi-source in DF cooperative networks with the PSR protocol based full-duplex energy harvesting over a Rayleigh fading channel: Performance analysis. Proceedings of the Estonian Academy of Sciences, 68(3), pp. 264-275.

5. TANG, K. and LIAO, S., 2020. Buffer-aided cooperative spectrum sharing with full-duplex wireless-powered relay. Computer Networks, 166.

6. TYAGI, A., SINGH, A. and BHULANIA, P., 2019. Partner Selection Based on Different Protocols in Cooperative Network by Using Nakagami-M Function, 2019 6th International Conference on Signal Processing and Integrated Networks, SPIN 20192019 , pp. 568-573.

7. FU, M. and WU, F., 2017. Investigation of multipath routing algorithms in software defined networking, Proceedings - 2017 International Conference on Green Informatics, ICGI 20172017 , pp. 269-273.

8. HAMZEH, H., HEMMATI, M. and SHIRMOHAMMADI, S., 2017. Priced-Based Fair Bandwidth Allocation for Networked Multimedia, Proceedings - 2017 IEEE International Symposium on Multimedia, ISM 2017 2017, pp. 19-24.

9. KHOSRAVI, M.R., BASRI, H. and ROSTAMI, H., 2018. Efficien routing for dense UWSNs with high-speed mobile nodes using spherical divisions. Journal of Supercomputing, 74(2), pp. 696-716.

10. LIN, Y., LIU, T., WANG, S. and LAI, Y., 2019. Proactive multipath routing with a predictive mechanism in software-defined networks. International Journal of Communication Systems, 32(14),

11. TANESSAKULWATTANA, S. and PORNAVALAI, C., 2019. Multipath energy balancing for clustered wireless sensor networks. Wireless Networks, 25(5), pp. 2537-2558.

12. ZAW, H.T. and MAW, A.H., 2019. Traffic management with elephant flow detection in software defined networks (SDN). International Journal of Electrical and Computer Engineering, 9(4), pp. 3203-3211.

13. FU, X., FORTINO, G., PACE, P., ALOI, G. and LI, W., 2020 Environment-fusion multipath routing protocol for wireless sensor networks. Information Fusion, 53, pp. 4-19.

\section{AUTHORS PROFILE}

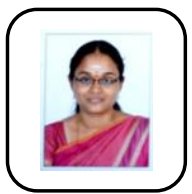

K.Shanmugapriya Assistant Professor Department of Computer Science \& Engineering, Bharath Institute of Higher Education and Research, Chennai, India

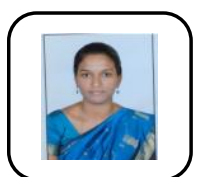

D.Jayapriya Assistant Professor, Department of Computer Science \& Engineering, Bharath Institute of Higher Education and Research, Chennai, India

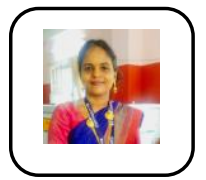

Kavitha G Assistant Professor, Department of Computer Science \& Engineering, Bharath Institute of Higher Education and Research, Chennai, India 